\title{
Quick Multicomponent Analysis Based on Matrix Isopotential Synchronous Fluorimetry
}

\author{
Géza Makkai and János Erostyák \\ Department of Experimental Physics, University of Pécs, Ifjúság u. 6., Pécs 7624, Hungary \\ Correspondence should be addressed to Géza Makkai, mgeza@fizika.ttk.pte.hu \\ Received 5 November 2012; Accepted 21 November 2012 \\ Academic Editors: M. L. Fernández de Córdova, L. Charbonnière, and S. Pandey
}

Copyright ( 2012 G. Makkai and J. Erostyák. This is an open access article distributed under the Creative Commons Attribution License, which permits unrestricted use, distribution, and reproduction in any medium, provided the original work is properly cited.

\begin{abstract}
Fast and precise methods are important in chemical and physical analysis, but determination of concentration of selected components in mixtures may have special difficulties. Excitation-emission matrices (EEM) are widely used in characterizing fluorescence emission. Based on these primary data, we present an improvement of matrix isopotential synchronous fluorimetry (MISF) and a method which ensures a very fast multicomponent analysis. MISF is generally combined with derivative technique (DMISF) to be able to eliminate the contribution of the background emission. Unfortunately, it is too sensitive to noises and fluctuations; thus the concentration region where it can be used, was limited until now. Our first aim was to reduce the influence of measurement errors and to increase the sensitivity of known MISF method by more than one order of magnitude. As a further result, using improved MISF, we got a method which ensures very fast determination of the wanted compound's concentration both in presence of one- or even two-component background of unknown concentration.
\end{abstract}

\section{Introduction}

The analysis of multicomponent mixtures without chemical or physical separation procedures is often based on spectroscopic measurements [1]. In conventional fluorescence spectroscopy, excitation and emission spectra are used for the analysis. In case of overlapping spectra, the synchronous fluorescence spectroscopy (SFS) [2-9] may help with its sharper and more separated spectral bands. Among the several variations of SFS and derivative SFS we will focus now on the matrix isopotential synchronous fluorimetry (MISF) [10-16]. When using MISF, one has to measure excitation-emission matrices (EEM). In an EEM there are fluorescence intensity data depending on both excitation and emission wavelengths. The neighboring spectral points of same fluorescence intensity form an isopotential trajectory in the EEM. The essence of MISF is that along the spectral points of an isopotential trajectory of a compound the fluorescence intensity of this compound is constant. In derivative MISF (DMISF) [17-19] the neighboring points on the isopotential scanning route are taken into account and subtracted from each other; thus the contribution of that compound is eliminated.

The aim of this work was to determine if a further improvement of MISF could be directly used as a fast, easy, and sensitive technique to quantify the concentration of compounds in the presence of known background compounds of unknown concentrations.

\section{Procedures and Methods}

The measured spectra always have systematic and random errors which disturb the precise investigation and judgment of a numerical method working with spectral data. To find the exact sensitivity of the improved MISF, we used simulated, noisy spectral data. Origin 7 was used to generate EEMs and Poisson-like noises and to calculate MISF spectra. Special algorithms and functions were built by our group in Origin C's environment. The fully detailed information about the procedures and methods used can be found in our previous work [14]. 


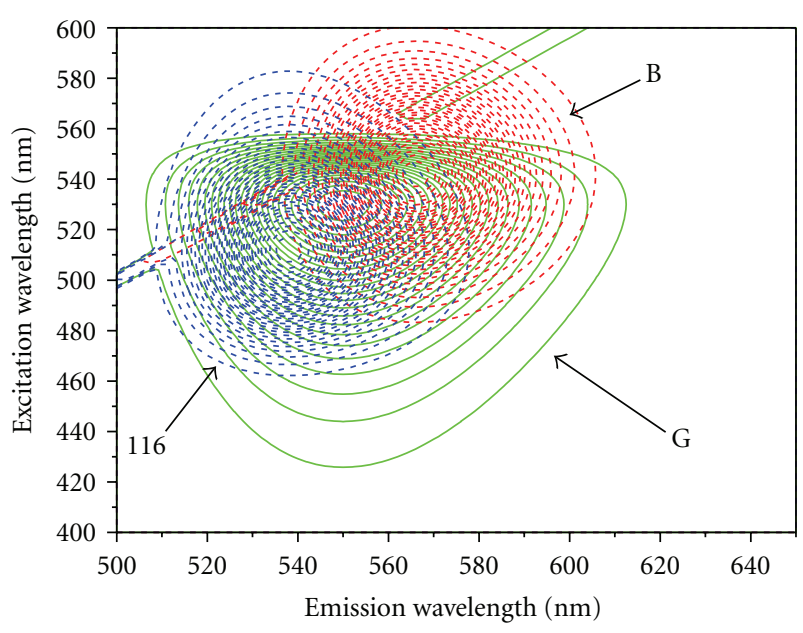

Figure 1: Contour plots of model components' EEMs. Simulating $10^{-7} \mathrm{M}$ concentration of each.

The main steps were:

(i) generating EEMs with predefined spectral shape (simulated the measured components concentration in the range of $\left.10^{-7} \mathrm{M}-10^{-11} \mathrm{M}\right)$ and noise level (\% percentage of the maximum intensity),

(ii) searching for the wanted compound's maximum intensity,

(iii) generating MISF points from the mixture's EEM at intensity level previously found,

(iv) getting intensity values from selected locations of MISF spectrum.

Figure 1 shows the contour plots of three spectrally strongly overlapping fluorescing components, similar, for example, to real compounds of Rhodamine 116, Rhodamine G, and Rhodamine B. For the sake of simplicity, hereinafter we will call the three model compounds as Component 116, Component $\mathrm{G}$, and Component $\mathrm{B}$.

\section{Results and Discussion}

Series of EEMs of two- and tree-component mixtures were analyzed. MISF spectra were constructed using isopotential trajectories (Figure 2) in the EEM of background sample. A precise and stable mathematical routine interpolating the three-dimensional data of EEM is needed to extract the proper excitation and emission wavelength parameters of a trajectory [12].

Figure 3 shows the 3D position of an MISF spectrum of the mixtures of $10^{-7} \mathrm{M}$ Component $\mathrm{B}$ as the wanted component and $10^{-7} \mathrm{M}$ Component $\mathrm{G}$ as the interfering one. The isopotential trajectories of the background component are projected to the $\lambda_{\text {exc }}-\lambda_{\text {emi }}$ plane. The points of MISF spectrum are highlighted in blue color over the orange wireframe of the mixture's EEM. A DMISF spectrum can be calculated as the difference of consecutive points of MISF spectrum. Unfortunately, it may suffer from the

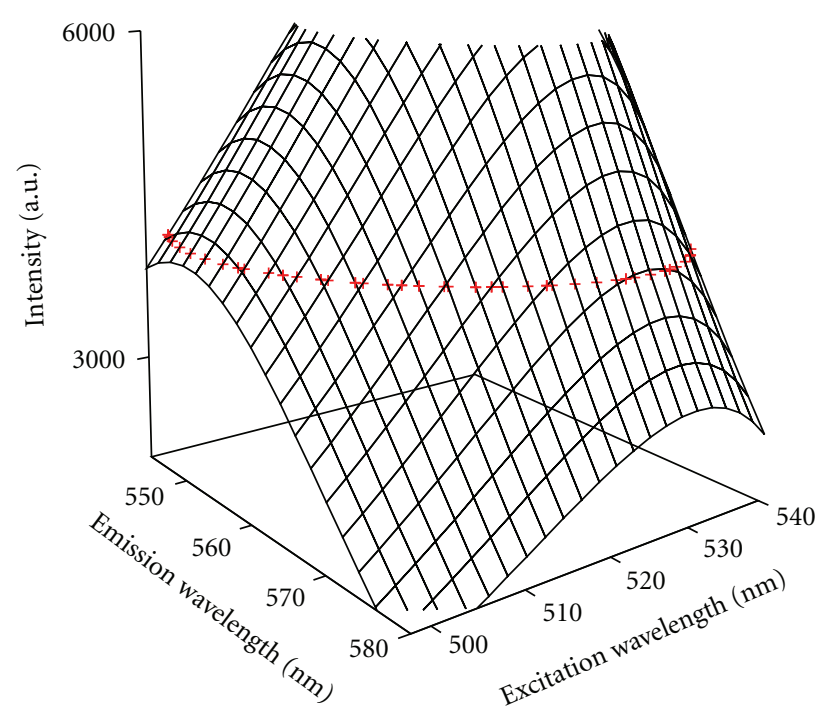

Figure 2: Grid points (red signs) of an isopotential trajectory at the level of Component B's maximum intensity on Component G.

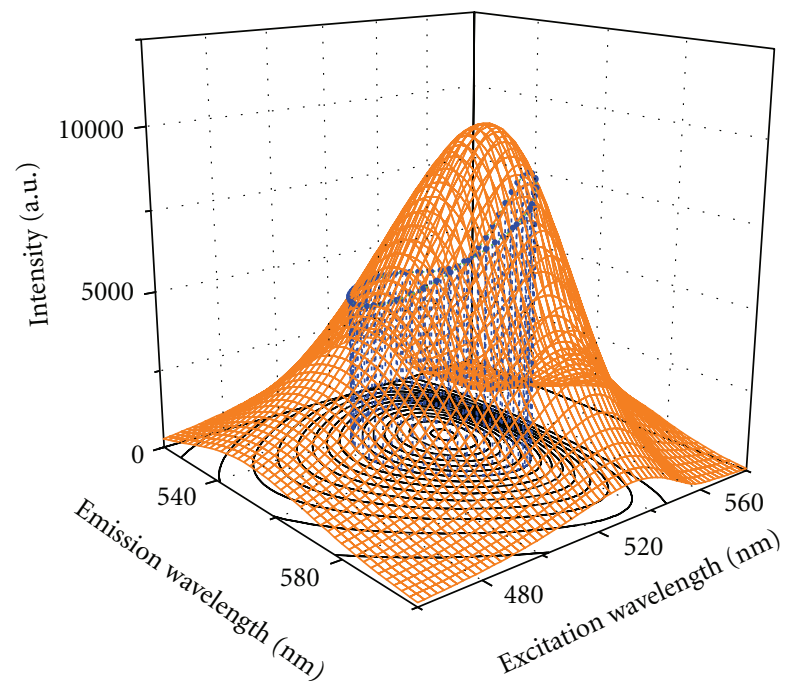

FIGURE 3: MISF points on mixture's 3D surface (blue signs on orange wireframe). Wanted component is $10^{-7} \mathrm{M} \mathrm{B}$, interfering one is $10^{-7} \mathrm{M} \mathrm{G}$. Bottom: contours of isopotential trajectories of background component.

unavoidable fluctuations of fluorescence intensity data, and already a relatively low noise of the background compound's fluorescence can defeat improving the sensitivity over $1: 20$.

3.1. Two-Component Mixtures. To step over this problem, we calculated intensity differences from not neighboring points of MISF spectrum. To reach the highest sensitivity we use the trajectory which passes through the fluorescence maximum of the wanted compound. At the selected points, the fluorescence intensity of the wanted component is significantly different, and this difference is much higher than the intensity fluctuations even in a noisy MISF spectrum. In Figure 4 the green trajectory of the background 


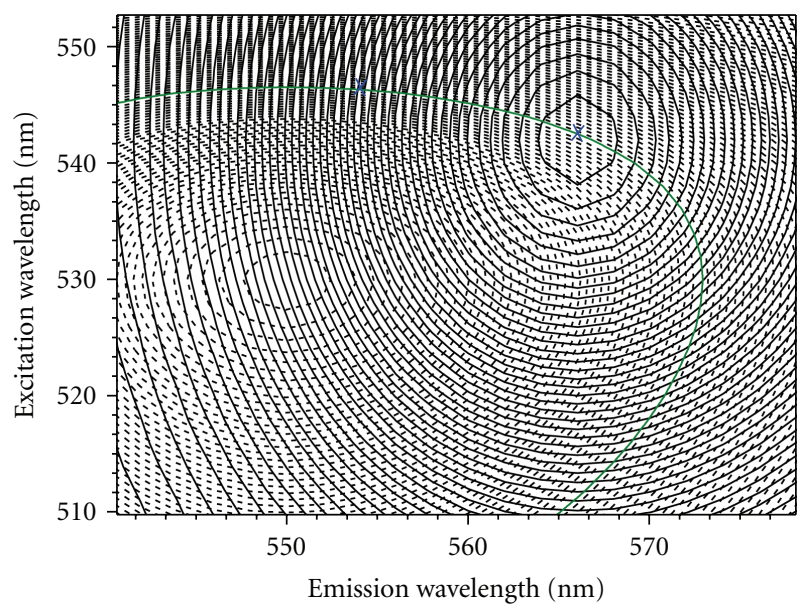

Figure 4: Isopotentials of Component G (background, dashed lines) and Component B (wanted, continuous lines) displayed in the same figure. Measuring points in case of these two interfering (one background) components are signed with blue crosses.

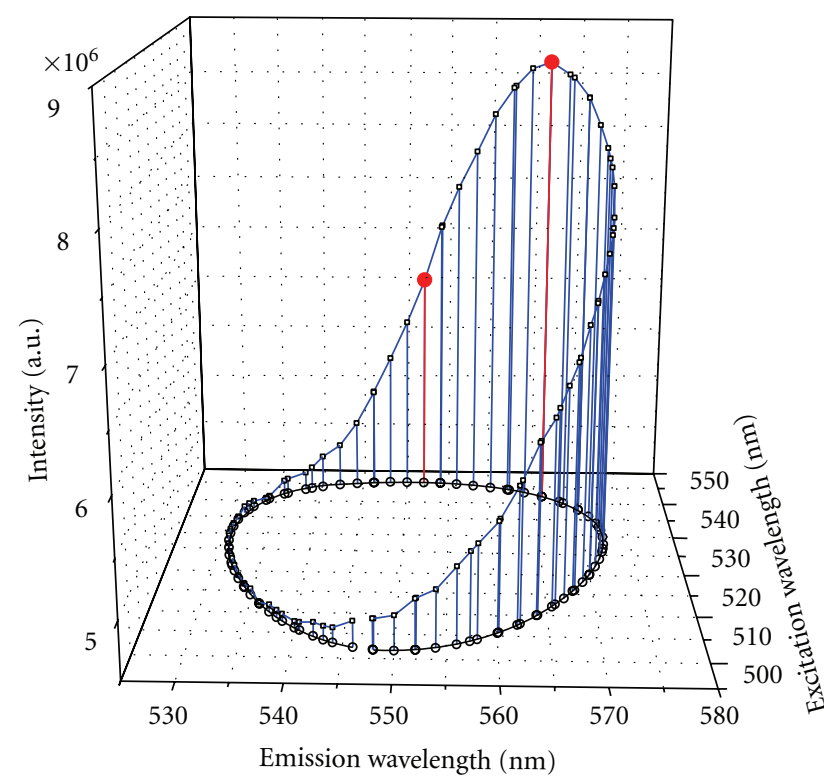

FIGURE 5: QMISF points (red signs) on calculated intensity values of MISF trajectory.

component is selected so that it goes through the intensity maximum of the wanted component. Blue crosses identify the spectral positions of the intensity maximum and one of the intensity half-maxima of the wanted component along the trajectory. Now, let us take the points of mixture's EEM using these spectral positions. Intensity difference between the two points is purely proportional to the concentration of the wanted component. Figure 5 shows the original MISF trajectory and the selected QMISF points.

3.2. Three-Component Mixtures. We extended this technique to three-component analysis, where the wanted compound (now Component 116) can be precisely found in the presence

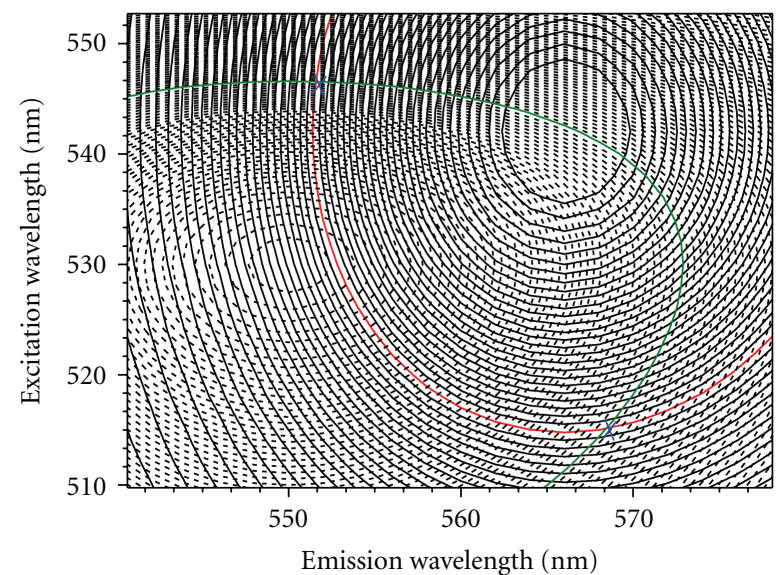

FIGURE 6: Measuring point pair in case of three interfering (two background) components (blue crosses). The isopotential trajectories of the two background components (Component $\mathrm{G}$ and Component B) are displayed.

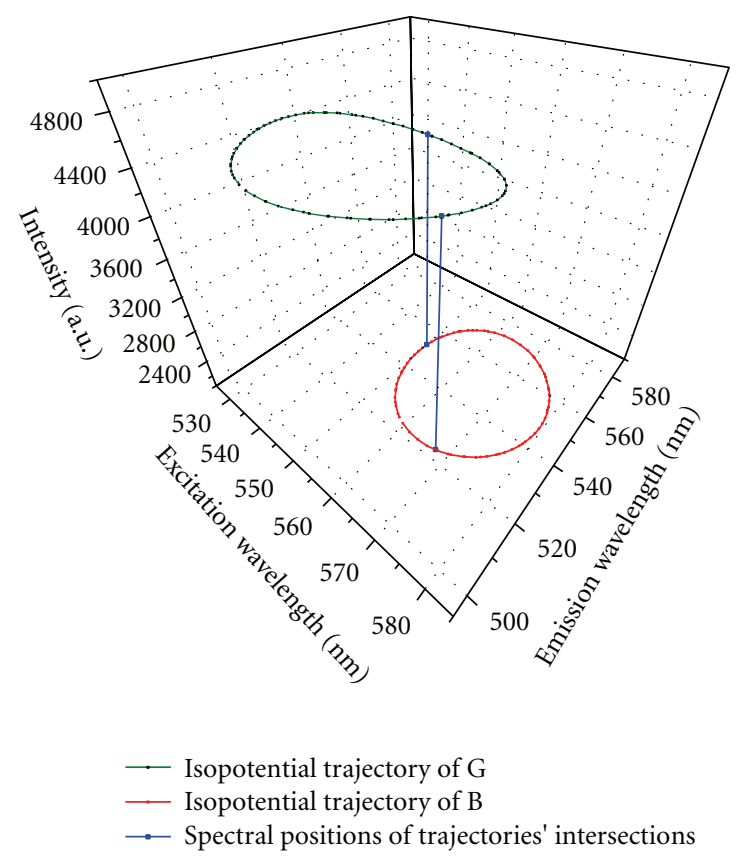

FiguRE 7: 3D visualization of virtually crossing trajectories and the selected spectral locations.

of two independently varying background components. To be able to do this, we have to find two trajectories which cross each other in the $\lambda_{\text {exc }}-\lambda_{\text {emi }}$ plane (green and red color trajectories belong to $10^{-7} \mathrm{M}$ Component $\mathrm{G}$ and $10^{-7} \mathrm{M}$ Component B background components, resp.). These two positions are marked by blue crosses in Figure 6 .

Figure 7 illustrates in 3D that the two trajectories lie at different intensity levels. Taking the intensity difference of the three-component mixture's EEM in the two selected spectral positions will eliminate influences of both background components. This intensity difference remains 


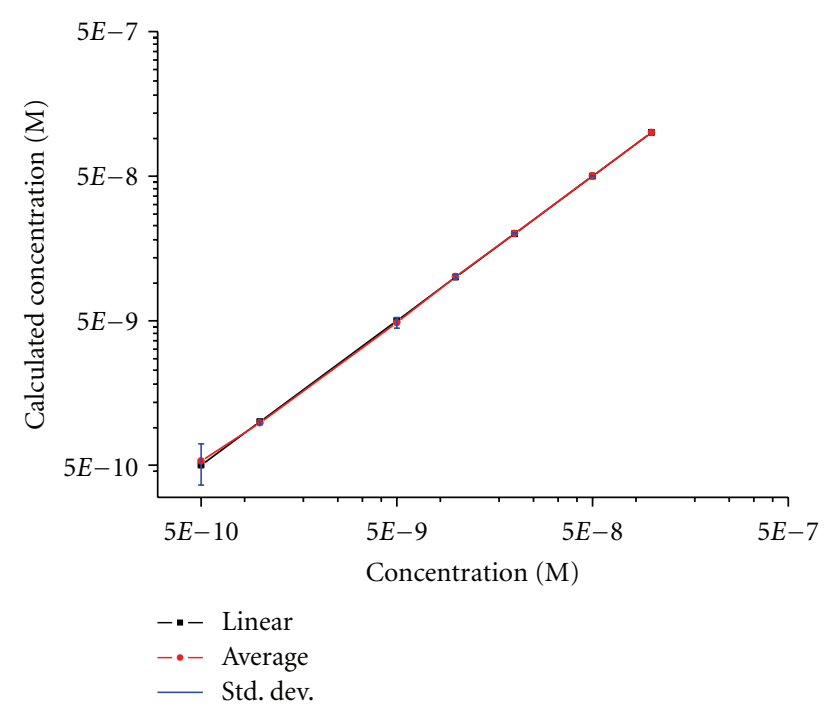

Figure 8: Linearity of findings in case of two components (constant $10^{-7} \mathrm{M}$ Model G, $5 * 10^{-10} \mathrm{M}-1 * 10^{-7} \mathrm{M}$ Model B). Noise level is $0.1 \%$.

proportional solely to the emission intensity of the wanted third component.

We found that two data points from a three-component mixture's EEM are enough to establish a reliable concentration determination of a wanted compound in the presence of two known background compounds of unknown concentration.

First, we have to know which compounds are in the solution, and precise measurement and analysis of components' EEMs are needed. But then, by selecting proper isopotential trajectories, the wanted component's concentration can be determined both in case of one-component or even twocomponent background of unknown concentration. We have to measure fluorescence intensity just in two spectral points, and the intensity difference $\left[I\left(\lambda_{\text {exc1 }}, \lambda_{\text {emi1 }}\right)-I\left(\lambda_{\text {exc } 2}, \lambda_{\text {emi2 }}\right)\right]$ is proportional to the concentration of the wanted compound.

This method is capable to quantitatively determine fluorophores being in less than $1: 200$ fluorescence intensity ratio compared to each other even at medium noise level ( $1 \%-2 \%$ of intensity of the background component). Even at $0.5 \%$ noise level a quantitative estimation can be done, where the level of noise added to the mixture randomly means the percentage of the maximum fluorescence intensity. Figures 8,9 , and 10 show the sensitivity of QMISF technique in case of $0.1 \%, 0.2 \%$, and $0.5 \%$ noise level. This statement is valid for both the two- and three-component variation of the improved MISF. Intensity fluctuations are shown on emission lines with different proportion of added noise on Figure 11.

\section{Conclusion}

QMISF is a significant (approximately two orders of magnitude) improvement compared to the classical DMISF method. At the final form, when the investigation of a certain

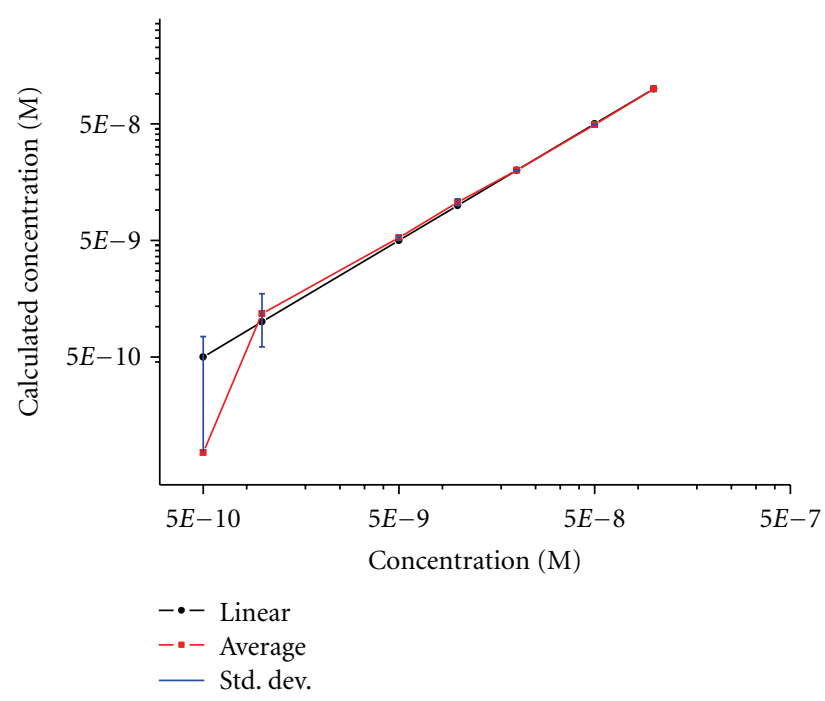

FIGURE 9: Linearity of findings in case of two components (constant $10^{-7} \mathrm{M}$ Model G, $5 * 10^{-10} \mathrm{M}-1 * 10^{-7} \mathrm{M}$ Model B). Noise level is $0.2 \%$.

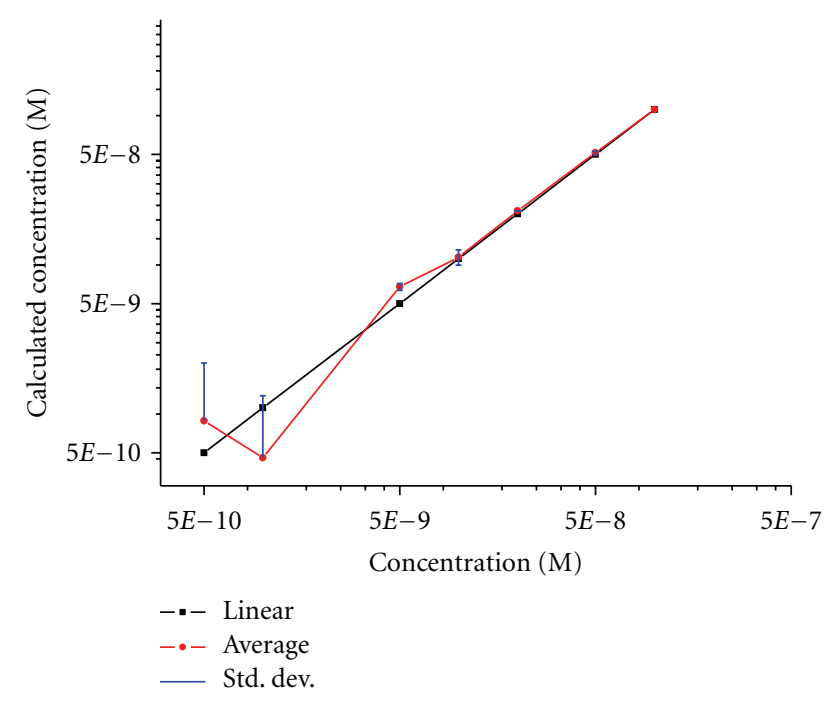

Figure 10: Linearity of findings in case of two components (constant $10^{-7} \mathrm{M}$ Model G, $5 * 10^{-10} \mathrm{M}-1 * 10^{-7} \mathrm{M}$ Model B). Noise level is $0.5 \%$.

complex sample has been done, only two spectral points are to be selected from the interpolated EEM. It means that a very sensitive, extremely fast, almost real time measurement can be established for determining the concentration of a wanted component very precisely. The most important features of the improved MISF are that

(i) the concentrations of background components are practically indifferent,

(ii) the sensitivity is much higher compared to classical DMISF,

(iii) measurements only at two spectral positions are enough. 


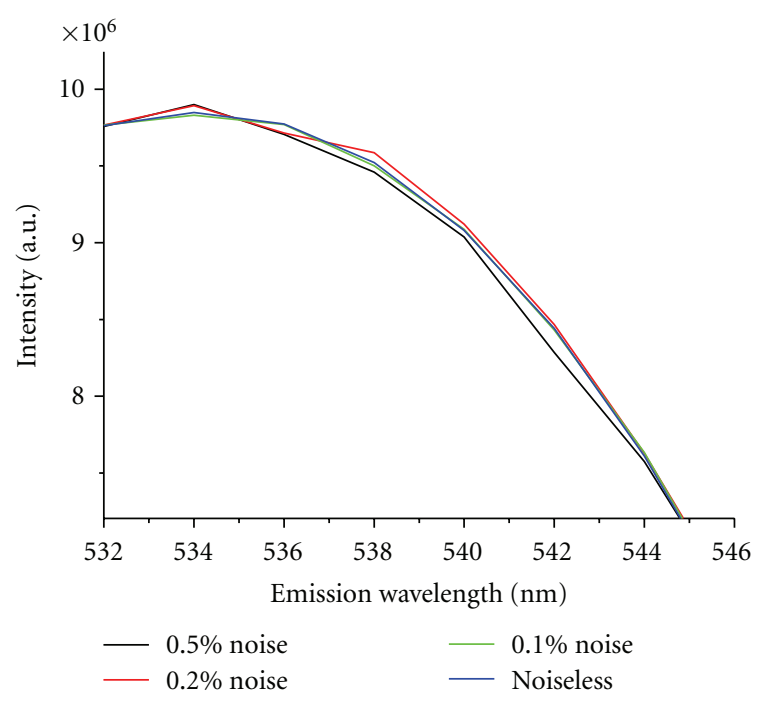

FIGURE 11: Comparison of the same simulated mixture's emission lines with different noise levels.

The $\mathrm{S} / \mathrm{N}$ ratio can be improved with increasing the integration time, the number of excitation pulses, the scanning time, and so forth in fluorescence measurements. A longer measuring time is still acceptable with the proposed new QMISF technique, when fluorescence intensity should be measured at only two spectral points in the routine measurements. Other techniques need measuring at much more spectral points, and there may be no time to collect these data to be noiseless enough.

A main force of QMISF is that the wanted component can be found at a reasonable measuring time at even $10^{-4}$ $10^{-3}$ relative concentration compared to the background (assuming similar quantum yields both for wanted and background components).

Based on these results, fast routine measurements could be implemented. Even simple fluorometers using narrowband interference filters could be built for special purposes. We are working on these new concepts and on further improvement of MISF.

\section{Acknowledgments}

The authors gratefully acknowledge the financial support from the Hungarian National Development Agency (SROP4.2.1.B-10/2/KONV-2010-0002 program). G. Makkai thanks the PhD School in Physics at the University of Pécs for further financial support.

\section{References}

[1] J. B. F. Lloyd, "Synchronized excitation of fluorescence emission spectra," Nature, vol. 231, no. 20, pp. 64-65, 1971.

[2] Á. Andrade-Eiroa, G. de-Armas, J. M. Estela, and V. Cerdà, "Critical approach to synchronous spectrofluorimetry. I," TrAC Trends in Analytical Chemistry, vol. 29, no. 8, pp. 885901, 2010.
[3] W. Zhang, D.-L. Lin, Z.-X. Zou, and Y.-Q. Li, "A novel approach for simultaneous determination of polycyclic aromatic hydrocarbons by Shpol'skii non-linear variable-angle synchronous fluorescence spectrometry," Talanta, vol. 71, no. 4, pp. 1481-1486, 2007.

[4] X. Liu, J. Jing, S. Li et al., "Measurement of pyrene in the gills of exposed fish using synchronous fluorescence spectroscopy," Chemosphere, vol. 86, no. 2, pp. 198-201, 2012.

[5] G. Hua, J. Broderick, K. T. Semple, K. Killham, and I. Singleton, "Rapid quantification of polycyclic aromatic hydrocarbons in hydroxypropyl- $\beta$-cyclodextrin (HPCD) soil extracts by synchronous fluorescence spectroscopy (SFS)," Environmental Pollution, vol. 148, no. 1, pp. 176-181, 2007.

[6] D. Patra and A. K. Mishra, "Investigation on simultaneous analysis of multicomponent polycyclic aromatic hydrocarbon mixtures in water samples: a simple synchronous fluorimetric method," Talanta, vol. 55, no. 1, pp. 143-153, 2001.

[7] M. A. Lage-Yusty, J. López-González, and J. Simal-Lozano, "Resolution of 13 polycyclic aromatic hydrocarbons by constant-wavelength synchronous spectrofluorometry," Analytical Sciences, vol. 21, no. 10, pp. 1203-1206, 2005.

[8] M. F. Pistonesi, M. S. Di Nezio, M. E. Centurión, M. E. Palomeque, A. G. Lista, and B. S. Fernández Band, "Determination of phenol, resorcinol and hydroquinone in air samples by synchronous fluorescence using partial leastsquares (PLS)," Talanta, vol. 69, no. 5, pp. 1265-1268, 2006.

[9] G. Hua, K. Killham, and I. Singleton, "Potential application of synchronous fluorescence spectroscopy to determine benzo[a]pyrene in soil extracts," Environmental Pollution, vol. 139, no. 2, pp. 272-278, 2006.

[10] J. A. Murillo Pulgarín, A. Alañón Molina, and I. SánchezFerrer Robles, "Simultaneous determination of two antiinflammatory drugs in serum using isopotential fluorimetry," Analytica Chimica Acta, vol. 625, no. 1, pp. 47-54, 2008.

[11] J. A. Murillo, A. Alañón, P. Fernández, and I. Sánchez-Ferrer Robles, "Direct determination of closely overlapping drug mixtures of diflunisal and salicylic acid in serum by means of derivative matrix isopotential synchronous fluorescence spectrometry," Analytica Chimica Acta, vol. 583, no. 1, pp. 5562, 2007.

[12] D.-L. Lin, L.-F. He, and Y.-Q. Li, "Rapid and simultaneous determination of coproporphyrin and protoporphyrin in feces by derivative matrix isopotential synchronous fluorescence spectrometry," Clinical Chemistry, vol. 50, no. 10, pp. 17971803, 2004.

[13] Y.-Q. Li, A. Abbas Falih Shindi, and X.-Y. Li, "Novel synchronous spectrofluorimetric techniques and their applications in the determination of diagnostically important compounds," CACS Communications, vol. 1, no. 4, pp. 18-22, 2010.

[14] G. Makkai, A. Buzády, and J. Erostyák, "Sensitivity test of derivative matrix isopotential synchronous fluorimetry and least squares fitting methods," Journal of Fluorescence, vol. 20, no. 1, pp. 87-94, 2010.

[15] Q. Liu, W. Huang, A. Abbas Falih Shindi, and Y.-Q. Li, "A novel rapid method for simultaneous determination of three diagnostically important porphyrins in erythrocytes using hyphenated synchronous fluorescence techniques," Talanta, vol. 88, pp. 663-668, 2012.

[16] A. Abbas Falih Shindi, P.-C. Zhou, Z.-X. Zou, and Y.-Q. Li, "A novel, selective, and rapid fluorimetric method for the simultaneous analysis of coproporphyrin and uroporphyrin in urine," Analytica Chimica Acta, vol. 664, no. 1, pp. 89-94, 2010. 
[17] X.-Y. Li, N. Li, H.-D. Luo et al., "A novel synchronous fluorescence spectroscopic approach for the rapid determination of three polycyclic aromatic hydrocarbons in tea with simple microwave-assisted pretreatment of sample," Journal of Agricultural and Food Chemistry, vol. 59, no. 11, pp. 58995905, 2011.

[18] E. Souri, M. Amanlou, H. Farsam, and A. Afshari, "A rapid derivative spectrophotometric method for simultaneous determination of naphazoline and antazoline in eye drops," Chemical and Pharmaceutical Bulletin, vol. 54, no. 1, pp. 119122, 2006.

[19] P.-C. Zhou, W. Huang, R.-B. Zhang et al., "A simple and rapid fluorimetric method for simultaneous determination of protoporphyrin IX and zinc protoporphyrin IX in whole blood," Applied Spectroscopy, vol. 62, no. 11, pp. 1268-1273, 2008. 


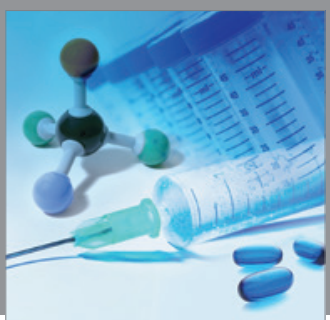

International Journal of

Medicinal Chemistry

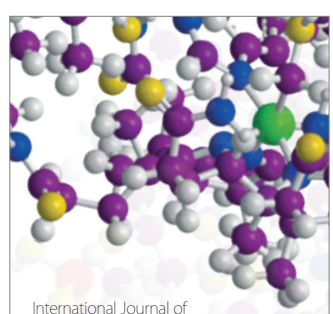

Carbohydrate Chemistry

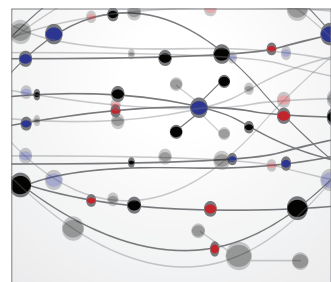

The Scientific World Journal
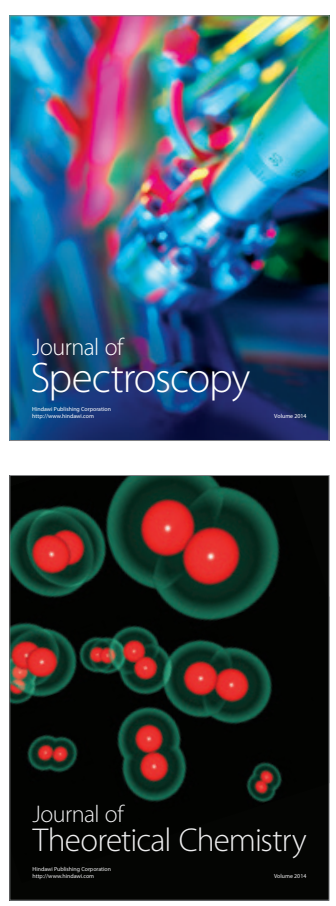
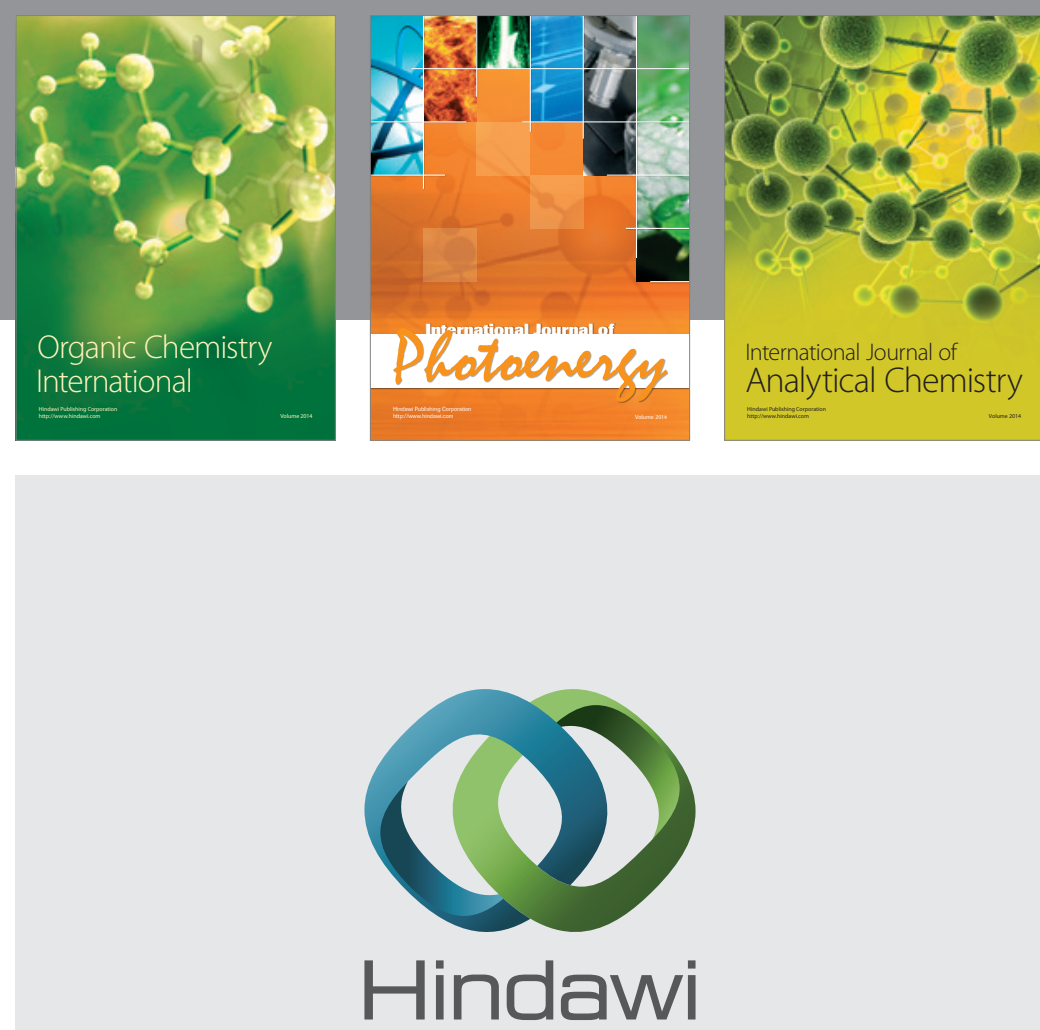

Submit your manuscripts at

http://www.hindawi.com
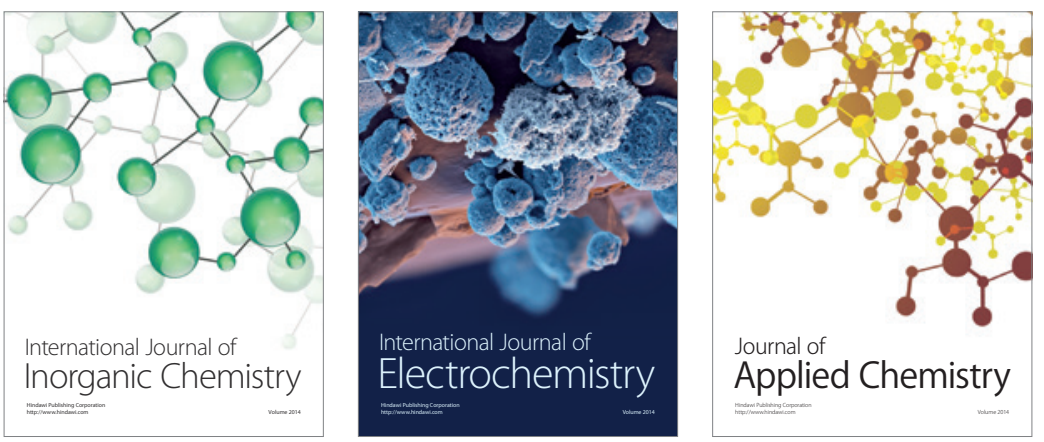

Journal of

Applied Chemistry
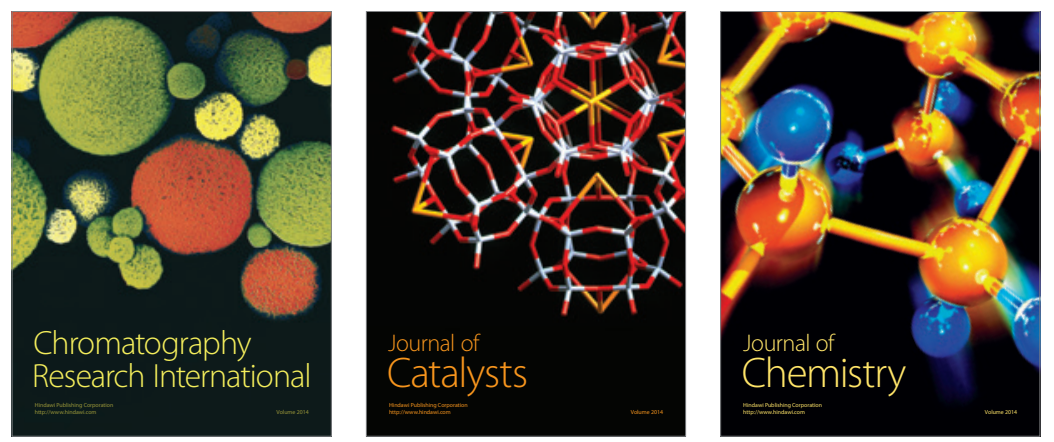
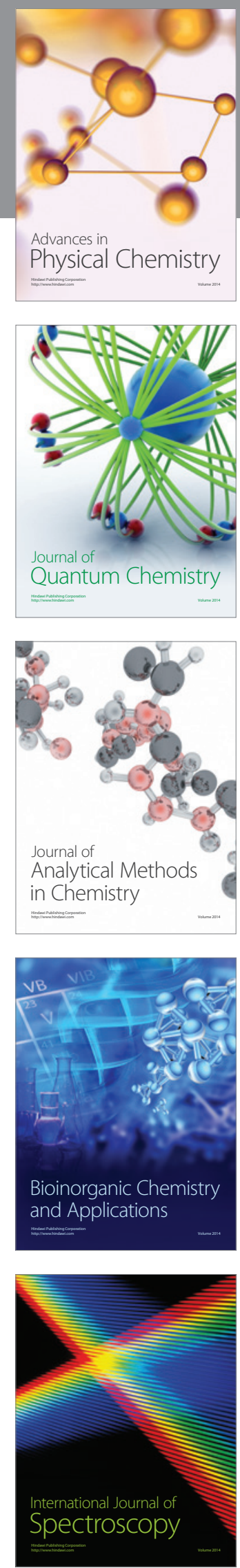